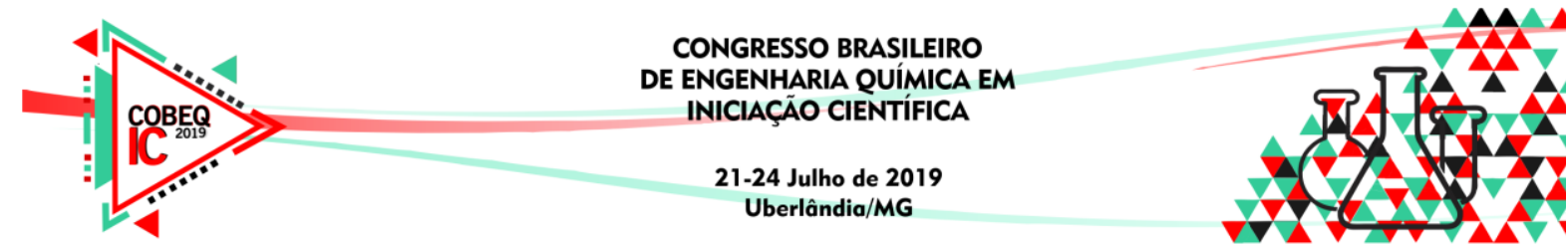

\title{
AVALIAÇÃO DE SUPORTES MAGNÉTICOS UTILIZADOS PARA IMOBILIZAÇÃO DA LIPASE DE CANDIDA RUGOSA
}

\author{
O. DOMINGUES ${ }^{1}$, L. K. SANTOS ${ }^{2}$, R. D. HERCULANO ${ }^{1}$, D. L. FLUMIGNAN ${ }^{3}$, e A. V. \\ PAULA $^{1}$ \\ ${ }^{1}$ Universidade Estadual Paulista, Departamento de Bioprocessos e Biotecnologia \\ ${ }^{2}$ Universidade Estadual Paulista, Departamento de Química Orgânica (CEMPEQC) \\ ${ }^{3}$ Instituto Federal de Educação, Ciência e Tecnologia de São Paulo (IFSP) \\ E-mail para contato: otavio.domingues@unesp.br, ariela.veloso@unesp.br
}

\begin{abstract}
RESUMO - Atualmente, o uso de nanopartículas magnéticas tem despertado grande interesse em processos industriais, e a combinação das suas propriedades juntamente com a imobilização de lipases tem sido desenvolvida a fim de se produzir suportes de fácil separação do meio reacional. Neste contexto, o objetivo do presente estudo foi imobilizar a lipase de Candida rugosa em nanopartículas magnéticas, tais como magnetita e maghemita, por adsorção física e por ligação covalente. Os biocatalisadores foram avaliados pela análise de espectroscopia de infravermelho (FTIR) e atividade hidrolítica. Dessa forma, a partir das análises realizadas, o melhor biocatalisador obtido foi o imobilizado por ligação covalente em maghemita, apresentando uma atividade hidrolítica de 174,67 U/g.
\end{abstract}

\section{INTRODUÇÃO}

No atual contexto industrial, a busca por formas de reaproveitamento de biocatalisadores têm ganhado amplo espaço dentre as pesquisas para melhoria e redução de custos em processos biotecnológicos, como na utilização das enzimas. Essas, são polímeros proteicos formados por ligações peptídicas entre dois aminoácidos adjacentes, apresentando uma região tridimensional formada por resíduos de aminoácidos (CAMPESTRINI et al., 2005). Dentre as enzimas, as lipases se destacam pela sua capacidade em realizar a hidrólise de triglicerídeos de cadeia longa, cujas aplicações industriais vão desde o uso na indústria alimentícia à obtenção de moléculas bioativas (REZANKA et al., 2017). Além disso, o uso deste biocatalisador mostra-se mais ecológico devido à menor produção de agentes tóxicos e subprodutos (ZHENG et al., 2017). Dentre as lipases oriundas de diversos microrganismos, o biocatalisador utilizado no presente trabalho foi a lipase de Candida rugosa.

Considerando o alto valor agregado e as vantagens do uso das lipases, a imobilização é uma alternativa para sua reutilização, influenciando e modificando parâmetros como a estabilidade após imobilizado, podendo ser realizada por diferentes metodologias (CARVALHO et al., 2015). A adesão por adsorção física ocorre pela simples interação da enzima com o suporte utilizado, sem a utilização de interações química no processo (SHELDON; PELT, 2013). Entretanto, a imobilização por meio da ligação covalente ocorre por uma forte ligação química entre a enzima e o suporte (TAN et al., 2010). Para o uso de um material como suporte para a imobilização, deve se considerar a superfície de contato, 


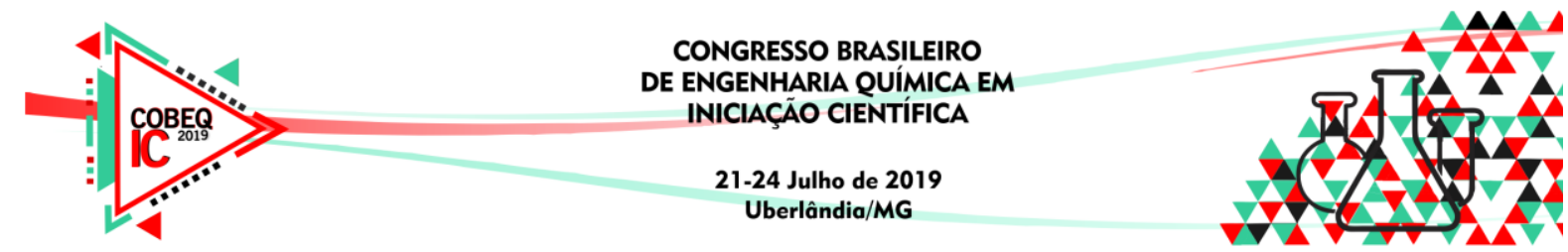

presença de íons, solubilidade, resistência mecânica, e o biocatalizador de interesse, sendo classificados quanto a sua composição, em inorgânicos e orgânicos, e estrutura morfológica, em não porosos, porosos e estrutura em gel (CARVALHO et al., 2015; BRÍGIDA, 2010).

Por isso, em virtude da busca por melhorias em processos biotecnológicos e a utilização de novos suportes para imobilização de enzimas, os suportes magnéticos chamam a atenção devido às vantagens como resistência a temperaturas elevadas, transferência de massa e a superfície disponível para a imobilização (PINTO, 2017). No presente trabalho, o suporte utilizado para a imobilização foram maghemita $\left(\gamma \mathrm{Fe}_{2} \mathrm{O}_{3}\right)$ e magnetita $\left(\mathrm{Fe}_{3} \mathrm{O}_{4}\right)$, óxidos de ferro formados por uma estrutura composta por íons de oxigênio, cujos espaços intersticiais estão preenchidos com por íons de ferro ferroso $\left(\mathrm{Fe}^{2+}\right)$, íons de ferro férrico $\left(\mathrm{Fe}^{3+}\right)$, se diferenciando pela ausência de ferro ferroso para a síntese de maghemita (DUNLOP \& ÖZDEMIR, 1997). No mais, a principal justificativa para a utilização de materiais magnéticas deve-se a fácil remoção do derivado imobilizado com um campo magnético após o final do processo.

\section{MATERIAIS E MÉTODOS}

\subsection{Síntese das nanopartículas magnéticas}

A síntese da magnetita foi realizada segundo a metodologia proposta por Mijone (2014), utilizando hidróxido de sódio e hidróxido de amônio como agente precipitante, e a maghemita segundo a metodologia modificada de Junior e seus colaboradores (2010).

\subsection{Modificação das nanopartículas e imobilização da lipase}

Para a imobilização por ligação covalente, fez-se necessária uma etapa de modificação das nanopartículas, sendo o processo de ativação, com APTES (3- aminopropiltrietoxisilano), como descrito por Kumar et al. (2013) e o processo de funcionalização, com glutaraldeído, como descrito por Vescovi et al. (2016). Assim, para a imobilização da lipase foram utilizadas as nanopartículas não modificadas, para a imobilização por adsorção física, e as nanopartículas modificadas, para a imobilização por ligação covalente, seguindo a metodologia modificada proposta por Paula (2012).

\subsection{Caracterização física do derivado imobilizado}

A fim de se analisar os processos de modificação e imobilização, foi realizada a espectroscopia de infravermelho (FTIR), utilizando o espectrômetro de infravermelho PLATINUM-ATR (Bruker). Os gráficos foram plotados utilizando o software ORIGIN 8.0.

\subsection{Determinação da atividade hidrolítica}

Para a determinação da atividade hidrolítica, foi utilizado o método titulométrico usando emulsão de azeite de oliva, de acordo com metodologia prevista por Paula (2012). 


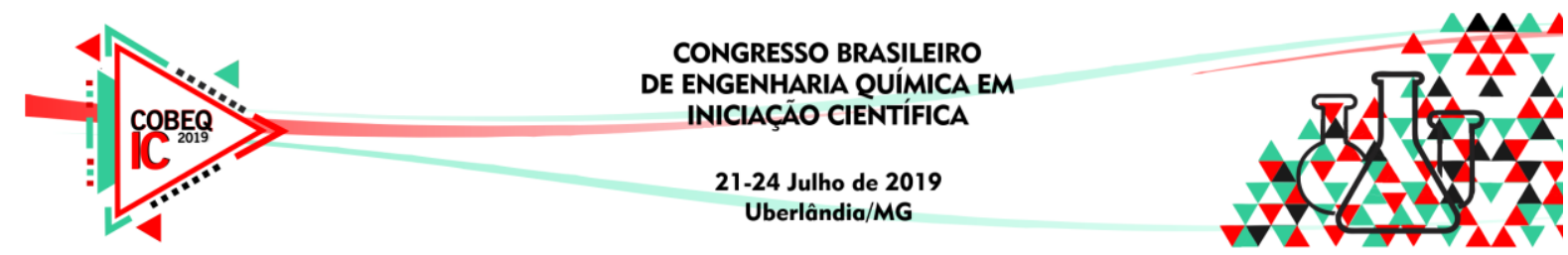

\section{RESULTADOS E DISCUSSÃO}

\subsection{Síntese das nanopartículas magnéticas}

Para a síntese da maghemita, utilizou-se e temperatura de $250^{\circ} \mathrm{C}$ ao invés dos $200^{\circ} \mathrm{C}$, sendo esta modificação necessária a fim de se evitar a inversão da estrutura da maghemita em hematita, considerando a sua temperatura de inversão ( $\mathrm{T}_{\text {inv }}$ ) mínima em $250^{\circ} \mathrm{C}$ (DUNLOP \& ÖZDEMIR, 1997). Dessa forma, partindo-se de 5,52 $\mathrm{g}$ de $\mathrm{FeCl}_{3}$ e 2,30 $\mathrm{g}$ de $\mathrm{FeCl}_{2}$ para a síntese da magnetita, obteve-se uma massa de 4,56 g e 4,63 g, rendimento de 58,31\% e $59,21 \%$, para as sínteses utilizando hidróxido de sódio e hidróxido de amônio, respectivamente. Já a síntese de maghemita, partindo-se de $10,03 \mathrm{~g}$ de $\mathrm{FeCl}_{3}$, foi obtida uma massa de $4,91 \mathrm{~g}$, rendimento de $49 \%$.

\subsection{Modificação das nanopartículas e imobilização da lipase}

A modificação foi realizada em parte das nanopartículas para a imobilização por ligação covalente, sendo o processo de ativação com (3-aminopropil)trietoxisilano (APTES), um reagente acoplador de silano e grupamentos aminos, e funcionalização com glutaraldeído, promovendo reações cruzadas irreversíveis e resultando em uma diferença de elétrons nos carbonos do grupo funcional carbonila, facilitando a interação entre a enzima e a nanopartículas modificada (LIANG et al., 2014; HAO et al., 2019; PEREIRA, 2009; NELSON \& COX, 2014). Abaixo, é mostrada na Figura 1 a espectroscopia em infravermelho dos suportes após o processo de modificação.

Figura 1 - Espectroscopia em infravermelho (FTIR): magnetita $(\sim \mathrm{NaOH})$ (vermelho), magnetita $(\sim \mathrm{NH} 4 \mathrm{OH})$ (azul) e maghemita (preto).

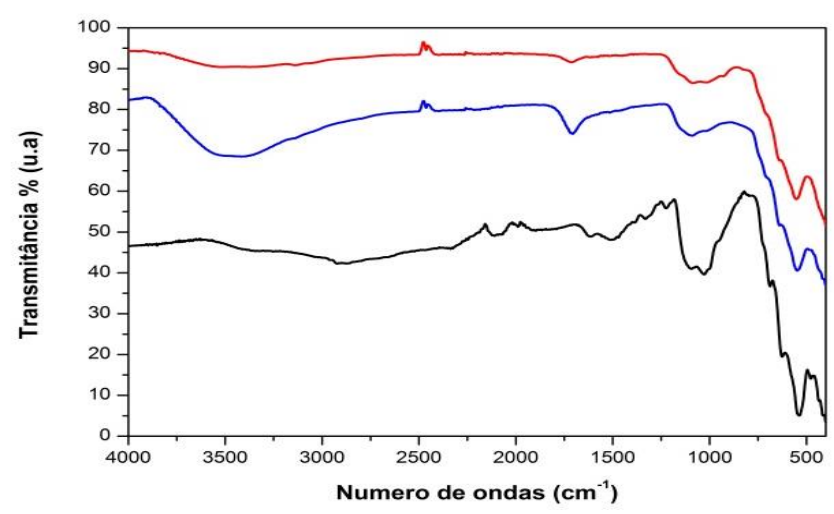

Por meio da análise da figura anterior, é possível observar regiões de estiramento de grupamentos característicos dos compostos utilizados para o processo de modificação, como grupamentos silano, alcóxi, metil e carbonila (CASAGRANDE \& REPETTE, 2018; PADILLA et al. 2011; PAVIA et al., 2010). Dessa forma, a lipase de Candida rugosa foi imobilizada nas nanopartículas por adsorção física e ligação covalente, utilizando as nanopartículas puras e modificadas, respectivamente. Assim, após a imobilização, foi realizada a caracterização física do derivado imobilizado por espectroscopia em infravermelho. A seguir, são mostradas as espectroscopias de infravermelho dos derivados imobilizados, sendo a Figura 2 referente a análise da imobilização por adsorção física e a Figura 3 da imobilização por ligação covalente. 


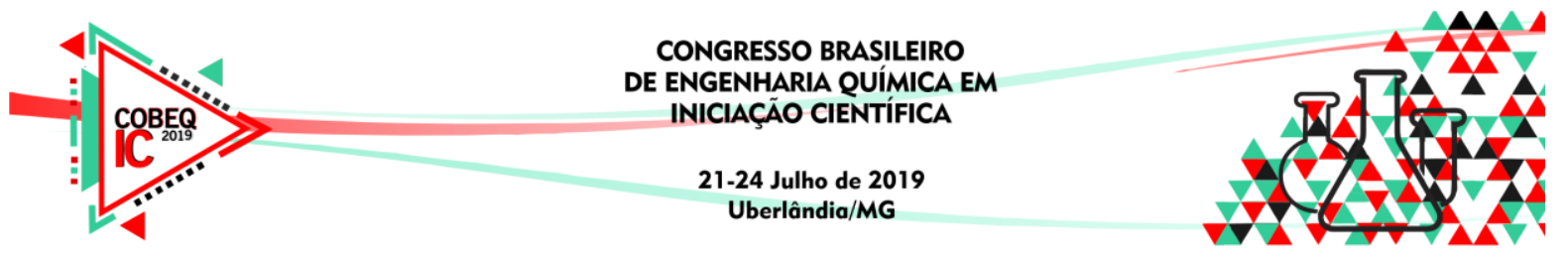

Figura 2 - Espectroscopia de infravermelho do derivado imobilizado por adsorção física: magnetita $(\sim \mathrm{NaOH})$ (vermelho), magnetita ${ }_{(\sim \mathrm{NH} 4 \mathrm{OH})}($ azul), maghemita (preto) e lipase (rosa).

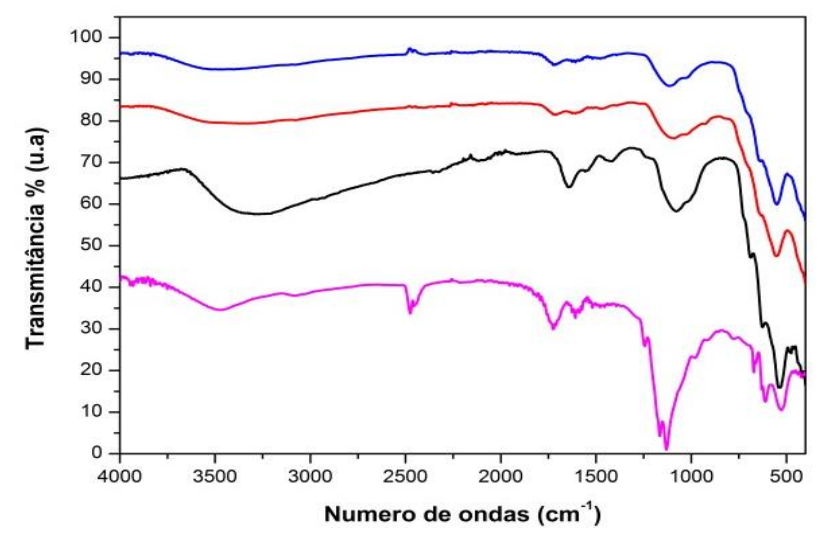

Figura 3 - Espectroscopia de infravermelho do derivado imobilizado por ligação covalente: magnetita $(\sim \mathrm{NaOH})$ (vermelho), magnetita $(\sim \mathrm{NH} 4 \mathrm{OH})$ (azul), maghemita (preto) e lipase (rosa).

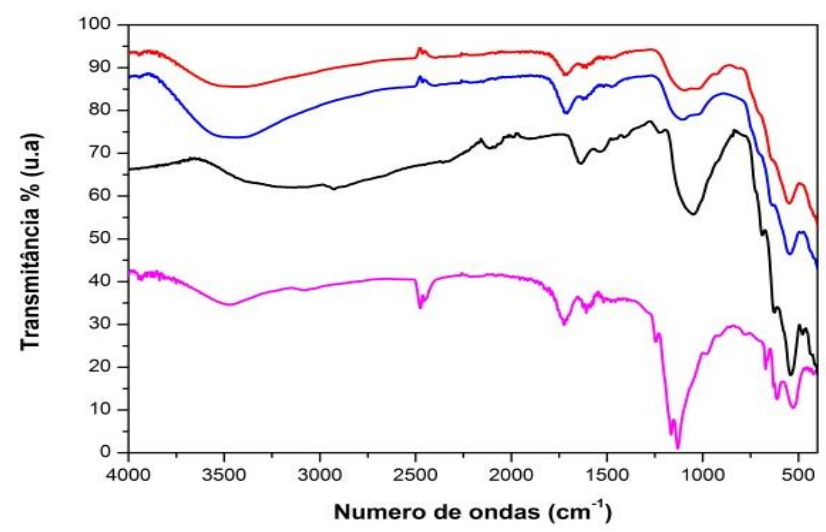

Assim, considerando a regiões identificadas nos gráficos com número de ondas característicos dos estiramentos de ligações presentes na lipase, como mostrado por Padilla et al. (2011), é possível verificar um maior estiramento em regiões de ligações características da lipase quando imobilizada por adsorção física e por ligação covalente em maghemita, uma vez que, estas foram as nanopartículas que apresentaram menores valores de transmitância nas regiões identificadas para o estiramento de grupamentos característicos das lipases.

\subsection{Determinação da atividade hidrolítica}

A partir dos dados de atividade hidrolítica contidos na Tabela 1 a seguir, é possível concluir que em ambos os processos de imobilização, os melhore valores de atividade foram obtidos para a utilização da maghemita como suporte de imobilização. Além disso, Jafarian et al. (2018) mostrou que a imobilização da lipase de Candida rugosa em nanofolhas de óxido de grafeno por ligação covalente resultou em uma atividade hidrolítica de 167,04 U/g. No mais, Hou et al. (2015) apresentou uma atividade de 119,43 U/g para a imobilização da lipase de Candida rugosa em concha magnética de polidopamina/alginato por ligação covalente. Nesse contexto, a pequena diferença encontrada entre os dados e a literatura pode ser justificada pela maior área superficial interna disposta em materiais porosos, favorecendo uma 


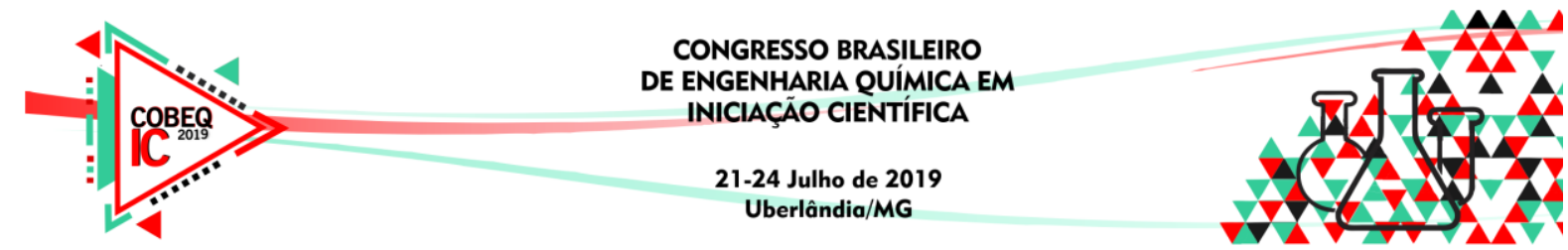

melhor utilização da estrutura para imobilização da enzima (BRÍGIDA, 2010). Dessa forma, os valores obtidos para o presente estudo estão próximos aos valores apresentados pela literatura para a utilização de suportes magnéticos, comprovando um bom aproveitamento das nanopartículas magnéticas como suporte para a imobilização da lipase.

Tabela 1 - Resultados de atividade hidrolítica médias (U/g) dos derivados imobilizados.

\begin{tabular}{|c|c|c|}
\hline \multirow{2}{*}{ Nanopartícula magnética } & \multicolumn{2}{|c|}{ Atividade hidrolítica (U/g) } \\
\cline { 2 - 3 } & Adsorção física & Ligação covalente \\
\hline \hline Magnetita $(\mathrm{NaOH})$ & $115,18 \pm 24,020$ & $144,35 \pm 12,81$ \\
\hline Magnetita $\left(\mathrm{NH}_{4} \mathrm{OH}\right)$ & $121,87 \pm 12,77$ & $144,89 \pm 12,05$ \\
\hline Maghemita & $126,37 \pm 19,66$ & $174,67 \pm 12,98$ \\
\hline
\end{tabular}

\section{CONCLUSÃO}

Partindo-se da síntese das nanopartículas magnéticas, o maior rendimento foi obtido para a síntese da magnetita sintetizada utilizando hidróxido de amônio como agente precipitante. No mais, quando analisadas as espectroscopias de infravermelho, verificou-se um maior estiramento de regiões características da lipase quando imobilizada em maghemita, tanto por adsorção física quanto por ligação covalente. Além disso, em relação à atividade hidrolítica, os melhores resultados foram também obtidos para a utilização da maghemita em ambos os processos de imobilização. Portanto, quando avaliada a utilização das nanopartículas magnéticas para a imobilização da lipase de Candida rugosa, verificou-se um melhor desempenho para a utilização da maghemita como suporte de imobilização.

\section{AGRADECIMENTOS}

À Fundação de Amparo à Pesquisa do Estado de São Paulo (FAPESP) - Processos 2017/11482-7 e 2018/09904-3.

\section{REFERÊNCIAS}

BRÍGIDA, A. I. S. Imobilização de lipases utilizando fibra de coco verde como suporte para aplicações industriais. 2010. 220 f. Tese (Doutorado em ciências) - Escola de Química, Universidade Federal do Rio de Janeiro, Rio de Janeiro, 2010.

CAMPESTRINI, E.; SILVA, V. T. M.; APPELT, M. D. Utilização de enzimas na alimentação animal, Revista Eletrônica Nutritime, v. 2, nº 6, p. 259-272, 2005.

CARVAlHO, N. B.; LIMA, A. S.; SOARES, C. M. F. Uso De Sílicas Modificadas Para Imobilização De Lipases. Química Nova, v. 38, n. 3, p. 399-409, Mar. 2015.

CASAGRANDE, C. A.; REPETTE, W. L. Monitoramento da hidrólise/condensação do tetraetoxisilano em solução com baixo teor alcoólico por análises de espectroscopia de infravermelho. Matéria - Rio de Janeiro, v. 23, 2018.DUNLOP, D. J.; ÖZDEMIR, Ö. Rock Magnetism, Fundamentals and Frontiers, Cambridge University Press, 1997.

HAO, Y.; CHENA, Y.; XIA, H.; GAO, Q. Surface chemical functionalization of starch nanocrystals modified by 3-aminopropyl triethoxysilane. International Journal of Biological Macromolecules, v. 126, p. 987-993, 2019.

HOU, C.; QI, Z.; ZHU, H. Preparation of core-shell magnetic polydopamine/alginate biocomposite for Candida rugosa lipase immobilization. Colloids and Surfaces B: Biointerfaces, v. 128, p. 544-551, 2015. 


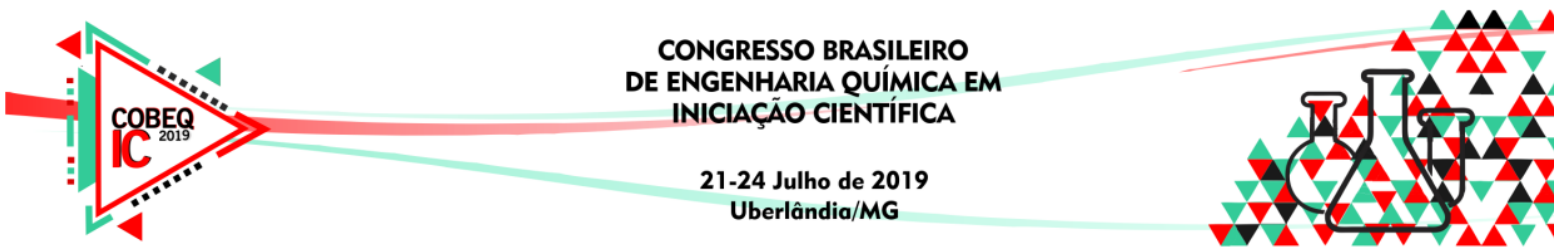

JAFARIAN, F.; BORDBAR, A. K.; ZARE, A.; KHOSROPOUR, A. The performance of immobilized Candida rugosa lipase on various surface modified graphene oxide nanosheets. International Journal of Biological Macromolecules, v. 111, p. 1166-1174, 2018.

JUNIOR, F. G. S.; MARINS, J. A.; RODRIGUES, C. H. M.; PINTO, J. C. A Magnetic Composite for Cleaning of Oil Spills on Water. Macromolecular Materials and Engineering, v. 295, n. 10, p. 942-948, 2010.

KUMAR, V.; JAHAN, F.; RAGHUWANSHI, S.; MAHAJAN, R. V.; SAXENA R. K. Immobilization of Rhizopus oryzae lipase on magnetic Fe3O4-chitosan beads and its potential in phenolic acids ester synthesis. Biotechnology and Bioprocess Engineering, v. 18, n. 4, p. 787-795, 2013.

LIANG, Y.; HUANG, J.; ZANG, P.; KIM, J.; HU, W. Molecular layer deposition of APTES on silicon nanowire biosensors: Surface characterization, stability and $\mathrm{pH}$ response, Applied Surface Science, 322 (2014) 202-208.

MIJONE, P. D. Imobilização de lipase em suporte magnetizado e aplicação na síntese de ésteres de etila em sistema descontínuo e contínuo. 86f. Dissertação (Mestrado na área de Processos Catalíticos e Biocatalíticos) do Programa de Pós-Graduação em Engenharia Química, Universidade de São Paulo, Lorena, 2014.NELSON, D. L.; COX, M. Lehninger - Princípios de Bioquímica, 6. ed. Porto Alegre: Artmed, 2014.

PADILLA, R. Y. C.; PENHA, J. M.; RICKS, A. T.; FRANCESCHI, E.; LIMA, A. S.; SILVA, D. P.; SOARES, C. M. F. Propriedades morfológicas e fisico-químicas da lipase Candida rugosa imobilizada em poli(3-hidroxibutirato-co-hidroxivalerato). Simpósio Nacional de Bioprocessos (SINAFERM), 2011.

PAULA, A. V. de. Reestruturação da gordura de leite por interesterificação enzimática empregando lipase imobilizada: otimização das condições reacionais e operacionais. 202 p. Tese (Doutorado em Biotecnologia Industrial na área de Microbiologia Aplicada). Universidade de São Paulo Escola de Engenharia de Lorena, Lorena, 2012.

PAULA, A. V.; MOREIRA, A. B. R.; BRAGA, L. P.; CASTRO, H. F.; BRUNO, L. M. Comparação do Desempenho da Lipase de Candida Rugosa Imobilizada em Suporte Híbrido de Polissiloxano-Polivinilálcool empregando Diferentes Metodologias. Quim. Nova, v. 31, n. 1, p. 35-40, 2008.

PAVIA, D. L.; LAMPMAN, G. M.; KRIZ, G. S.; VYVYAN, J. R. Introdução à Espectroscopia. Cengage Learning, 700 p., 2010.

PEREIRA, A. V. P. Análise e caracterização de tecidos porcino e bovino fixados com glutaraldeído para aplicação em biopróteses cardíacas. Dissertação: Mestrado. 2009.

PINTO, P. A. Imobilização de lipase em suporte magnetizado e aplicação na síntese de ésteres de etila em sistema descontínuo e contínuo 2017. 70f. Monografia de graduação em Engenharia Química - Escola de Engenharia de Lorena, Universidade de São Paulo, Lorena, 2017.

REZANKA, T.; PÁDROVÁ, K.; SIGLER, K. Regioisomeric and enantiomeric analysis of triacylglycerols. Analytical Biochemistry, v. 524, p. 3-12, May 2017.

SHELDON, R. A.; PELT, S. Enzyme immobilisation in biocatalysis: why, what and how. Chemical Society Reviews, v. 42, n. 15, 2013.

TAN, T.; LU, J.K; NIE, K.L; DENG, L.; WANG, F., Biodiesel production with immobilized lipase: A review. Biotechnology Advances, v.28, p.628-634, 2010.

VESCOVI, V.; KOPPA, W.; GUISÁN, J. M.; GIORDANO, R. L. C.; MENDES, A. A.; TARDIOLI, P. W. Improved catalytic properties of Candida antarctica lipase B multi-attached on tailormade hydrophobic silica containing octyl and multifunctional amino- glutaraldehyde spacer arms. Process Biochemistry, 2016.

ZHENG, M.; WANG, S.; XIANG, X.; SHI, J.; HUANG, J.; DENG, Q.; HUANG, F.; XIAO, J. Facile preparation of magnetic carbon nanotubes-immobilized lipase for highly efficient synthesis of 1,3-dioleoyl-2-palmitoylglycerol-rich human milk fat substitutes. Food chemistry, v. 228, p. 476-483, 2017. 